\title{
Development of a Real Time Supported Program for Motorbike Drivers Using Smartphone Built-in Sensors
}

\author{
Pham Van Thanh ${ }^{1,4}$, Tien-Anh Nguyen ${ }^{2}$, Nghia Tran Duc ${ }^{3}$, Nguyen Duc Anh ${ }^{4}$, Tran Duc-Tan ${ }^{1 *}$ \\ 1VNU University of Engineering and Technology (VNU-UET), Hanoi, Vietnam \\ phamvanthanh1209@gmail.com, tantd@vnu.edu.vn \\ 2Le Quy Don Technical University, Hanoi, Vietnam \\ anhnt007@gmail.com \\ 3Institute of Information Technology, VAST, Viet Nam \\ nghiatranduc1986@yahoo.com \\ 4The University of Fire Fighting and Prevention, Hanoi, Vietnam \\ anhpchp@gmail.com
}

\begin{abstract}
Using mobile phones during traffic progress is one of the main causes traffic accidents because drivers do not focus on driving, they try to listen phone calls or to text some messages... Most of research work has focused to car driving. However, using motorbike is very popular in some developing countries such as Vietnam, India, etc. Up to now, there are just a few works, which focus to motorbike driving with obvious limitations. Thus, in this research, we proposed a complete solution for bikers who own a smartphone. Our work exploits the information from built-in sensors in Android smartphone. A complete scheme for motorbike driving is proposed. In this scheme, the user state is detected by improving the current Google activity recognition API. If the state is "On vehicle", the phone automatically switches to silent mode and send to the caller an SMS. Our work provides a mechanism to receive the calls from VIP contacts and urgent calls. The phone would switch back to the normal mode if the state is not "On vehicle". Furthermore, it sends the accident location to the relatives when an accident occurs to save their lives automatically. The application was tested carefully and it can be used to protect the lives of motorbike drivers.
\end{abstract}

Keyword-3-DOF Accelerometers, GPS, Accidental Location, Motor Safe

\section{INTRODUCTION}

According to the Traffic Police Department, Vietnam has more than 45 million motorbikes in over 90 million people until 2016 with 21.568 traffic accidents occurred in this year and the number of people died and injured were 8.680 and 19.280 respectively (see Fig.1). One of the main causes in traffic accidents is using the phone while controlling vehicles (see Fig. 2). They usually use the phone for listening to music, calling, messaging or playing some app games like Pokemon Go...[3][4]. Hence, they lose focus on controlling vehicles because of being limited visibility and being distracted from other drivers... Then they cannot handle all situations. Furthermore, the time between the occurrence of accidents and the notification to relatives and medical services is too slow; it results in increasing the number of fatal cases [5].

In order to reduce the number of traffic accidents and people died as well, there are several published methods used to protect drivers during the controlling vehicles process in recent years by researchers and companies. Nevertheless, most of the reported publications focus on developing the supported systems for cars such as: Accelerometer based Transportation System [6], Accident Avoidance and Driver Assist Technologies [7], PRE-SAFE ${ }^{8}[8]$, etc. The systems in [11][12][13] used smartphones to detect the accident in combination with automatic sending alert notification to relatives and hospital services, but these applications do not have function to switch the phone to silent mode when receiving incoming calls from unimportant or unknown people while driving. Therefore, drivers are easily distracted while driving. 


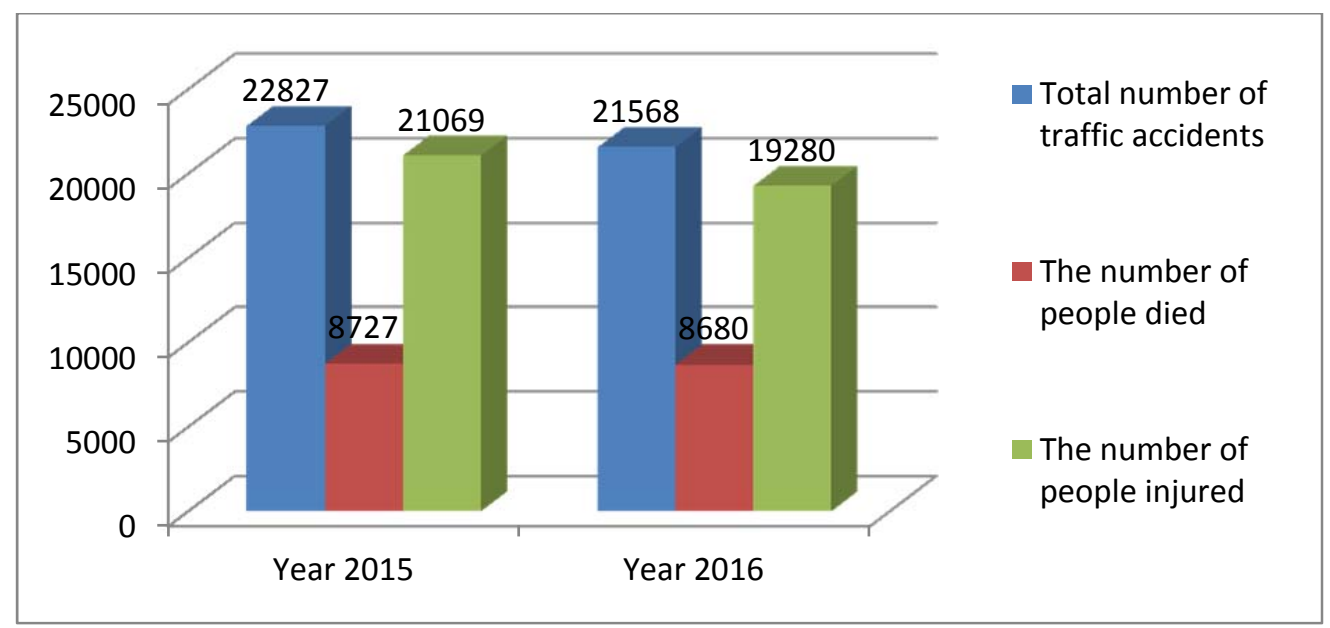

Fig. 1. The total number of traffic accidents, people died and people injured in 2015 and 2016 in Vietnam [1]

The private software S-Bike of Samsung was installed in Galaxy J 2017 series products to support for motorbike drivers during the driving process. S-Bike can filt all incoming calls and it also allows to receive the calls from important numbers and to answer the phone when drivers stopped. However, S-Bike also has some limitations that cannot change between silent mode and normal mode automatically, it needs to do manually. Hence, the phones are still in silent mode when drivers stopped, then they can lose calls or messages. Furthermore, S-Bike does not have the function that can send out messages or call relatives and hospital services when the accident occurred.

Based on the above limitations, this paper proposed to develop an efficient application that can be installed in smartphones to protect drivers' lives while driving motorcycle. The application will automatically recognize states of the user, if drivers are detected "On vehicle", the phone will automatically change to silent mode and all incoming calls from unimportant or unknown people will be rejected with a notification message "I'm driving, call me later" except the calls from VIP or urgent calls. If drivers got accident, a message that attached their position will be sent to relatives and hospital services.
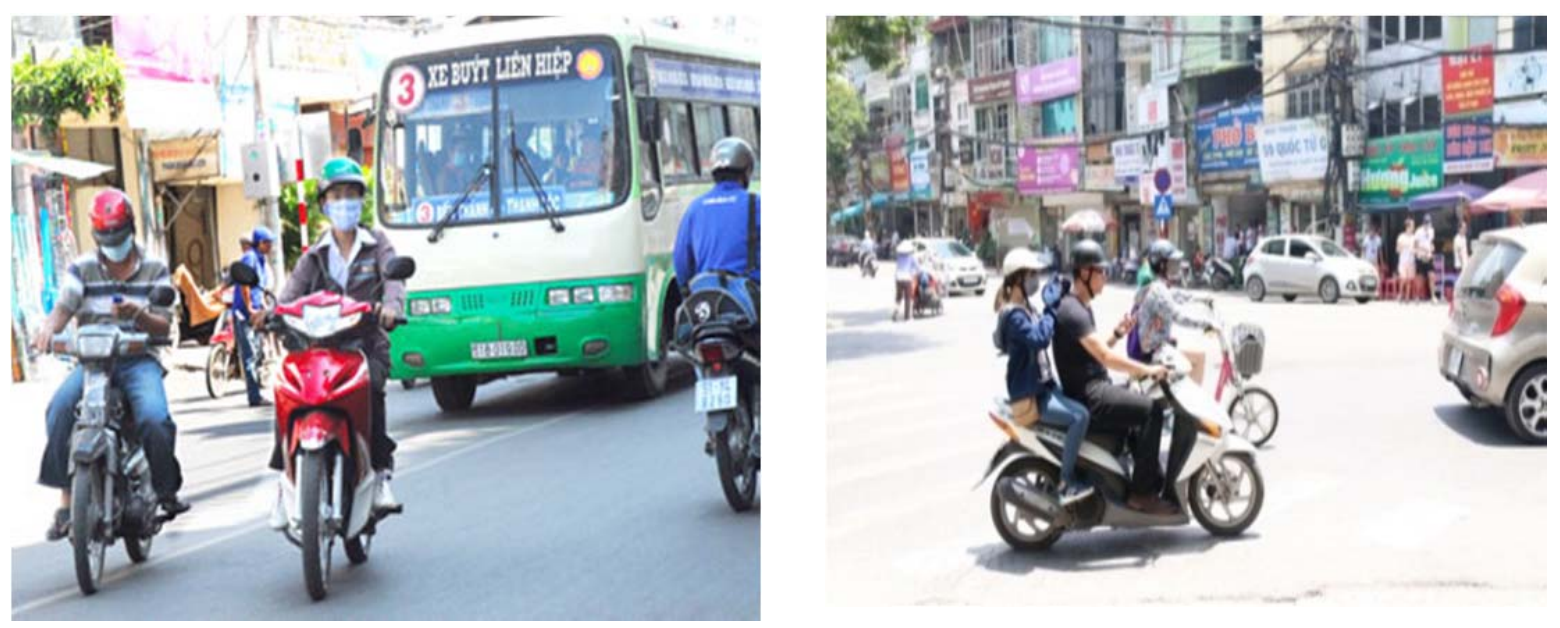

Fig. 2. Drivers are using a mobile phone while driving[3][4]

\section{METHODS}

\section{A. Hardware Components}

The prototype uses a computer with Microsoft ${ }^{\circledR}$ Windows ${ }^{\circledR} 10,64$ bit; Intel Core i5 Haswell 4200U 1.60 $\mathrm{GHz}$; $8 \mathrm{~Gb}$ of RAM and NVIDIA Geforce $720 \mathrm{M}$ as well. It also uses smartphone Samsung Galaxy Trend Plus GT S7580 for experiment testing with specifications as: - CPU: Dual core 1.2GHz; - RAM: $768 \mathrm{MB}$; - Android OS: Android 4.2.2 (Jelly Bean); - ChipSet: Broadcom BCM21664; - MicroUSB 2.0; - Sensor: Accelerometer (BOSCH BMC150), Proximity (GP2A), Magnetic (BOSCH BMC150).

\section{B. Software Components}

Android is an open source nature, which developed by Google based on the Linux kernel. It attracted a large community of developers with primarily designed for touchscreen mobile devices such as smartphones and tablet with specialized user interfaces for Android TV, Android Auto and Android Wear... Therefore, it is very 
popular in the number of mobile applications and mobile platform. Google APIs (Application Programming Interfaces) developed by Google allows communicating with Google Services such as Search, Gmail, Translate, Google Maps... The third-party apps can use Google APIs to take advantage or extend the functions of the existing services. Google activity recognition API (GAR_API) used machine learning classifiers to recognize user activities deployed as part of Google Play Services (see Fig. 3). Currently, it can detect 8 states of user include: IN_VEHICLE, ON_BICYCLE, ON_FOOT, RUNNING, STILL, TITLING, UNKNOWN, WALKING. Each state has a constant value as in Table 1.

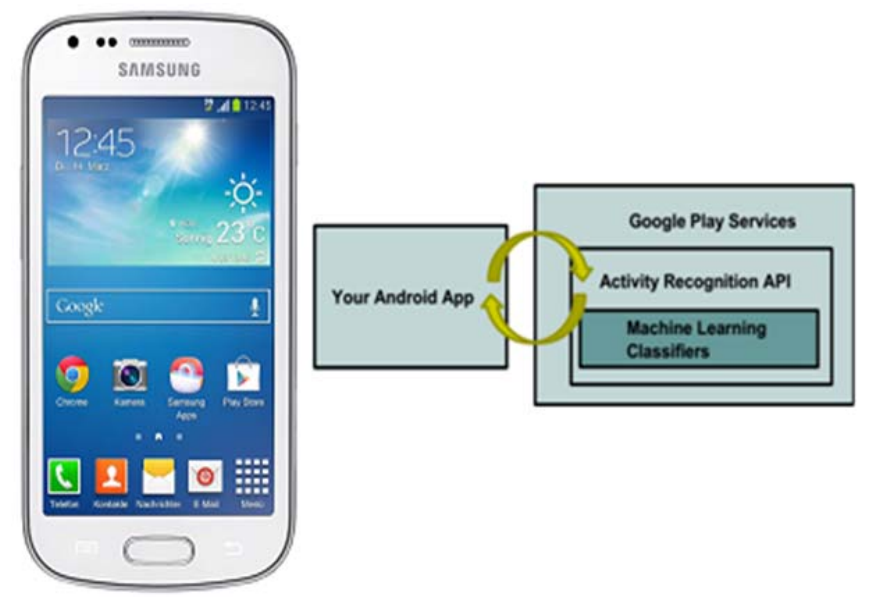

Fig.3. Google activity recognition API deployed as part of Google Play Services [19]

\section{The flowchart of the proposed work}

The flowchart of the proposed program is shown in Fig. 7. After installing this application on Android smartphone, the user click on "Start" button, and built-in accelerometers will start to acquire accelerations in the $\mathrm{X}, \mathrm{Y}$ and $\mathrm{Z}$ axes automatically. Consequently, two parallel threads will be executed.

In the first thread, if the driver is detected with the status of "On vehicle", the phone will be changed to silent mode. In this mode:

+ All normal phone calls will be rejected, and the caller will receive a designed message "I'm driving, call me later".

+ If the caller is in the VIP list, it allows the phone ringing and vibrating. The program will check the current velocity of the motorbike, and the motorbike driver can only answer the call only if he/she stops driving (i.e. the velocity is smaller than a determined threshold).

+ If the caller is not in the VIP list, but it is an emergency situation, the program will recognize it is an emergency call when the number of the phone calls is higher 3 times in 5 minutes. Similar to the scenario of the VIP call, in order to save the lives of drivers, this application requires he/she stop driving before answering the phone.

If the driver stops driving, the program will recognize this status and switches the phone to the normal mode. It means that he/she can hear the ring and the vibration of the phone call as usual. The flexible switching between "On vehicle" and "Not On Vehicle" will maximize the safety and the convenience of the motorbike drivers.

In the second thread, the phone monitors if the accident happens. In the case of an accident, an alert will notify the driver to check if he/she can manage this situation by himself/herself. After a period of time, it will send a message that attached the accident location to their relatives and hospital services. 


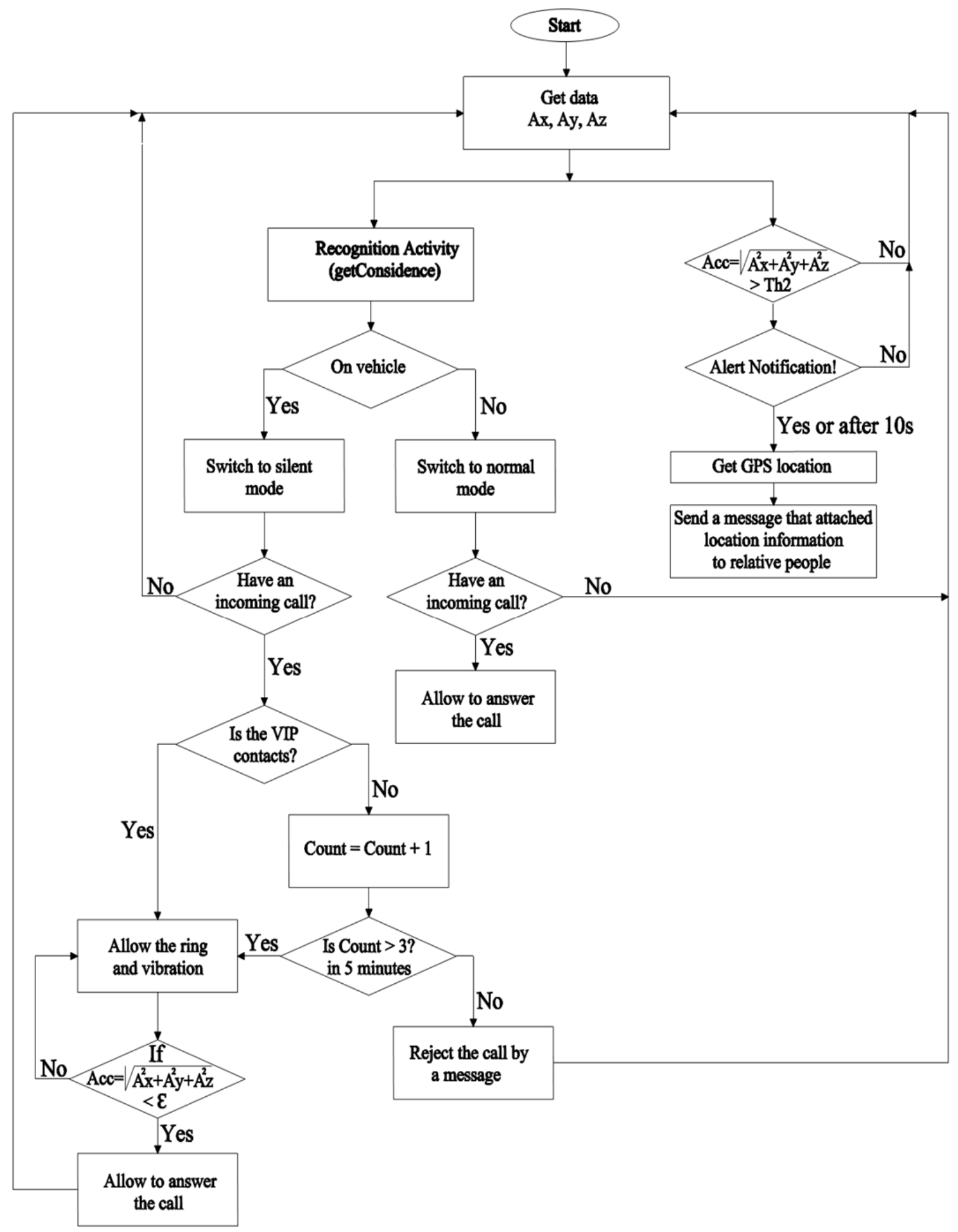

Fig. 4. The algorithm of the proposed system

1) Detecting the user's status

Activity Recognition is a Google service that allows android app developers detect activities of the users through the ActivityRecognitionAPI. In order to detect the status of a user, Google Play Services Activity Recognition API gives the ability to detect a number of our physical activities as in table 1 . All these activities can be detected simply by using an API to access Google Play Service. Detected Activity is obtained from ActivityRecognitionAPI. Some constant values showed as in the table below: 
TABLE I. The constants in Detected Activity[9]:

\begin{tabular}{|c|l|c|}
\hline Constants & \multicolumn{1}{|c|}{ Describe } & $\begin{array}{c}\text { Constant } \\
\text { values }\end{array}$ \\
\hline ON_VEHICLE & The device is in a vehicle, such as a motorbike, car, etc. & 0 \\
\hline ON_BICYCLE & The device is on a bicycle. & 1 \\
\hline ON_FOOT & The device is on a user who is walking or running. & 3 \\
\hline RUNNING & $\begin{array}{l}\text { The device is on a user who is running. This is a sub-activity of } \\
\text { ON_FOOT. }\end{array}$ & 4 \\
\hline STILL & The device is still (not moving). & 5 \\
\hline TITLING & $\begin{array}{l}\text { The device angle relative to gravity changed significantly. This often } \\
\text { occurs when a device is picked up from a desk or a user who is sitting } \\
\text { stands up. }\end{array}$ & 6 \\
\hline UNKNOWN & Unable to detect the current activity. & 7 \\
\hline WALKING & $\begin{array}{l}\text { The device is on a user who is walking. This is a sub-activity of } \\
\text { ON_FOOT. }\end{array}$ & \\
\hline
\end{tabular}

The using Google ActivityRecognitionAPI is also exiting some limitations because of traffic conditions and people habits. Then, there are some types of "On vehicle" samples that Google ActivityRecognitionAPI detected the wrong state.

In this paper, we proposed cascade algorithm and threshold value (Th1) to improve the accuracy of Google ActivityRecognitionAPI in detecting "On vehicle" state. Th1 was chosen based on the result of analysis carefully on our recorded data. Hence, threshold Th1 and cascade algorithm play an essential role in distinguishing between "On vehicle" and other states. If after two consecutive times of checking in 1 second, the results are "On vehicle", the final decision will be "On vehicle" and vice versa. Nevertheless, if the results of two consecutive times are different, we propose an algorithm requires to check more times and compares with Th1 to improve the accuracy of the system as in table 2:

TABLE II. The final decision of recognition process using Cascade algorithm

\begin{tabular}{|c|c|c|}
\hline $\mathbf{1}^{\text {st }}$ time & $\mathbf{2}^{\text {nd }}$ time & Final Decision \\
\hline On vehicle & On vehicle & On vehicle \\
\hline On vehicle & Not On vehicle & Need to check more times \\
\hline Not On vehicle & On vehicle & Need to check more times \\
\hline Not On vehicle & Not On vehicle & Not On vehicle \\
\hline
\end{tabular}

In the case of detecting user status is driving, the authors had developed a function that called "blockCall()", it will automatically change smartphone to silent mode and send out a message with content: "I'm driving, call me later" to the caller if they receive incoming call while driving except the calls from VIP contacts. This is a very essential function that does not lose focus on driving of the user.

\section{2) Detecting accidents}

3-DOF sensor used to get accelerations in 3 axis $\mathrm{X}, \mathrm{Y}$ and $\mathrm{Z}$ (see Fig. 5); the $\mathrm{X}$ axis is horizontal and point to right; the $\mathrm{Y}$ axis is vertical and points up and the $\mathrm{Z}$ axis points toward the outside of the screen.

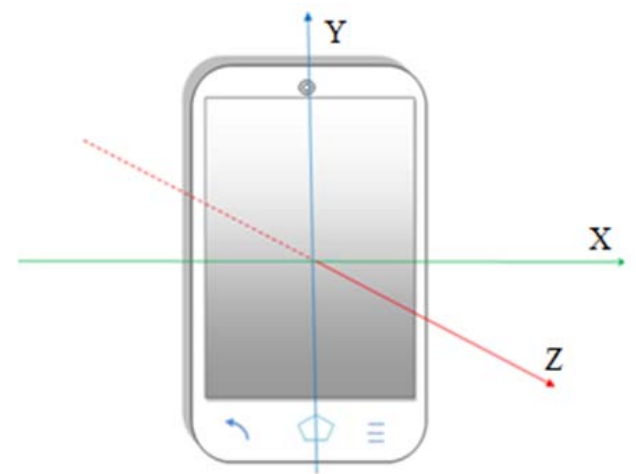

Fig. 5. The system coordinates 
Android allows to specify the sampling frequency using one of four sensor delay:

- SENSOR_DELAY_FASTEST: 0 millisecond delay

- SENSOR_DELAY_GAME: 20 milliseconds delay

- SENSOR_DELAY_NORMAL: 200 milliseconds delay

- SENSOR_DELAY_UI: 60 milliseconds delay

An acceleration sensor determines the values by measuring the forces that are applied to the sensor as the following relationship:

$$
\operatorname{Ad}=-\frac{\sum \mathrm{F}_{\mathrm{s}}}{\text { mass }}
$$

However, the force of gravity is always influencing the measured acceleration according to the following relationship:

$$
\mathrm{A}_{\mathrm{d}}=-\mathrm{g}-\frac{\sum \mathrm{F}_{\mathrm{s}}}{\text { mass }}
$$

When the device is located on a table, the accelerometer reads a magnitude of $g=9.81 \mathrm{~m} / \mathrm{s}^{2}$. Similarly, when the device is in free fall, acceleration magnitude values change rapidly and a low-pass filter was used to isolate the force of gravity.

Figure 6 shows the processing of acceleration to determine accident:

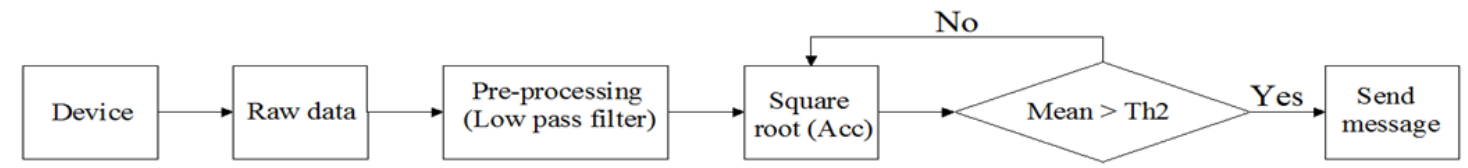

Fig. 6. The processing of acceleration data

After pre-process by using a low - pass filter, the square root (Acc) is calculated:

$$
A c c=\sqrt{A_{X}^{2}+A_{Y}^{2}+A_{Z}^{2}}\left(m / s^{2}\right)
$$

where: $A_{X}, A_{Y}, A_{Z}$ are acceleration value in each axis.

The mean obtained by using a moving-average filter, this is represented by the following difference equation:

$$
\mathrm{y}(\mathrm{n})=\frac{1}{\text { windowSize }}(\mathrm{x}(\mathrm{n})+\mathrm{x}(\mathrm{n}-1)+\cdots+\mathrm{x}(\mathrm{n}-(\text { windowSize }-1)))
$$

where $y(n)$ is "mean"; windowSize is the size of window in processing.

By comparing the mean with threshold $\mathrm{Th} 2=4 \times \mathrm{g}$ (with $\mathrm{g}=9.81 \mathrm{~m} / \mathrm{s}^{2}$ ) [6] to detect an accident. If an accident occurs, an automatic message that includes the address of the accident will be sent to relative members and medical services.

\section{III.RESULTS AND DISCUSSION}

For the experimental testing, we carried out on 5 smartphones Samsung Galaxy Trend Plus GT S7580 and 17 students (12 males and 5 females) ages: 19-21, height: $1.56-1.75 \mathrm{~m}$, weight: $46-65 \mathrm{~kg}$ who were randomly selected from various students in University of Engineering and Technology, Vietnam National University, Hanoi.

\section{A. Detecting the user's status}

Google Activity Recognition API is used to detect if the driver's status is "On Vehicle" or not. The Fig. 7 and Fig. 8 show the result of training "Walking" (i.e. the user was actually walking in the street). In Fig.7, it can be seen that the device is successful to determine the status of "Walking" with the percentage varied from $92 \%$ to $100 \%$. Also in this test, Fig. 8 shows that the percentage of "On Vehicle" is very small (from $0 \%$ to $8 \%$ ).

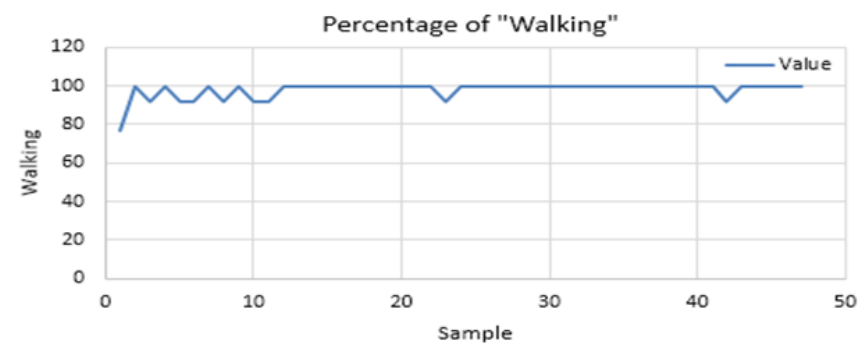

Fig. 7. The percentage of detecting "Walking" in training "Walking" 


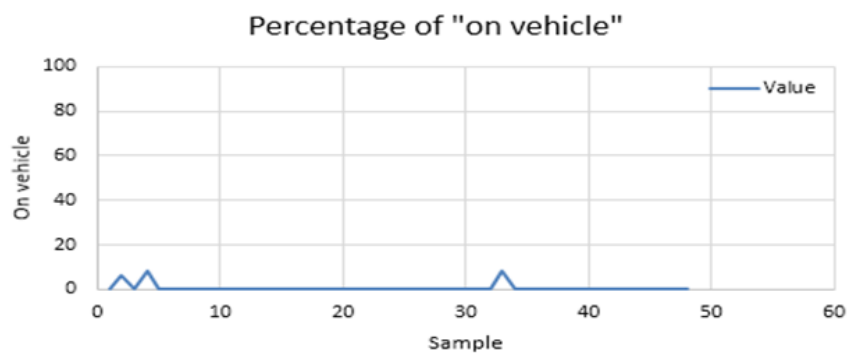

Fig. 8. The percentage of detecting "On vehicle" in training "Walking"

Figures 9 and 10 show the result of training "On vehicle" with the speed which varied from $20 \mathrm{~km} / \mathrm{h}$ to 40 $\mathrm{km} / \mathrm{h}$ in the distance of $2 \mathrm{~km}$. The device is successful to determine the status of the users with the percentage of "On vehicle" (i.e. from $38 \%$ to $100 \%$ ) is always larger than that of "Walking" (i.e. from $0 \%$ to $23 \%$ ). Nevertheless, most of values in "On vehicle" are bigger than $60 \%$. Hence, the threshold chosen to detect "On vehicle" is Th1 $=60 \%$.

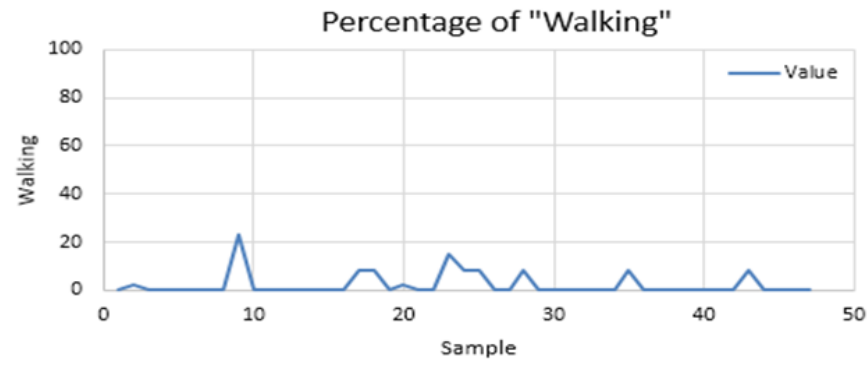

Fig. 9. The percentage of detecting "Walking" in training "On vehicle"

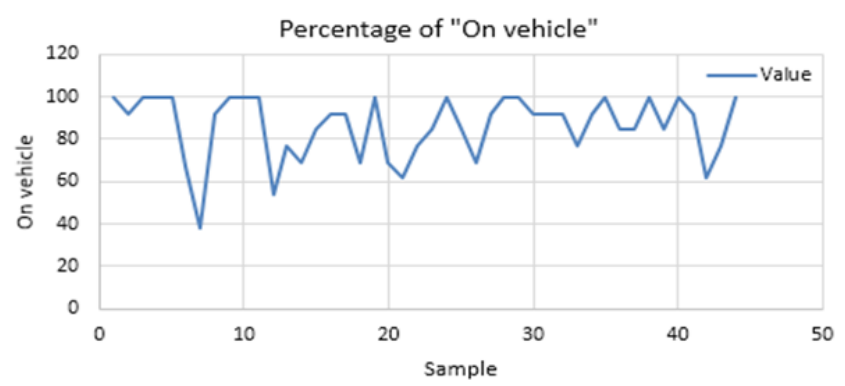

Fig. 10. The percentage of detecting "On vehicle" in training "On vehicle"

When the users set up our application, they can create VIP contacts as shown in Fig. 11. It will allow the phone to ring and vibrate when receiving an incoming call in spite of driving motorcycle. Regular calls will automatically rejected because the phone is changed to silent mode, and the caller will receive an auto replying message.

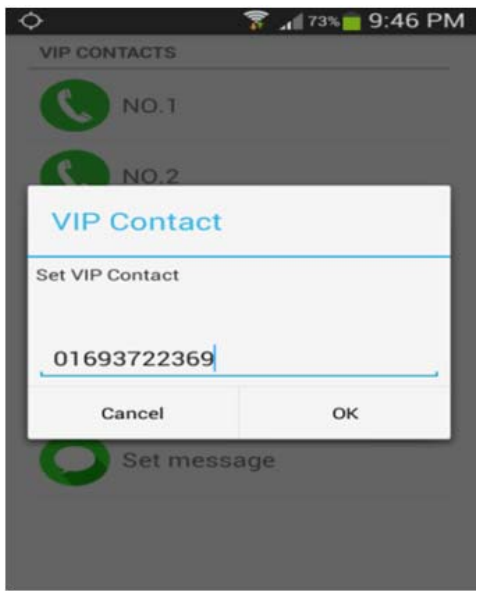

a)

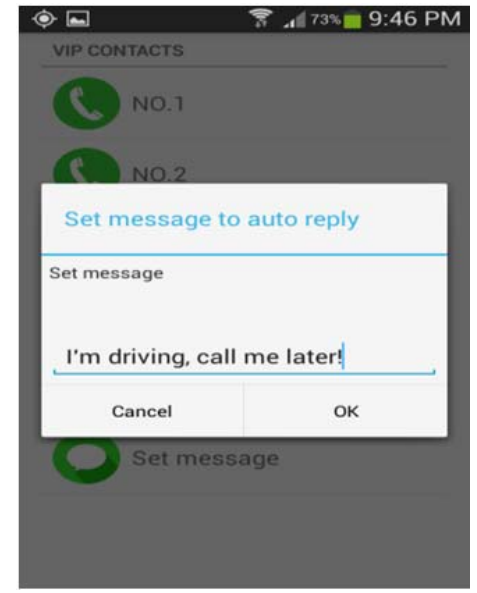

b)

Figure 11. (a) Setting the VIP contacts; (b) Setting message 
Figure 12 shows that the phone automatically detected the status of users and change the phone to silent mode when the users was driving.
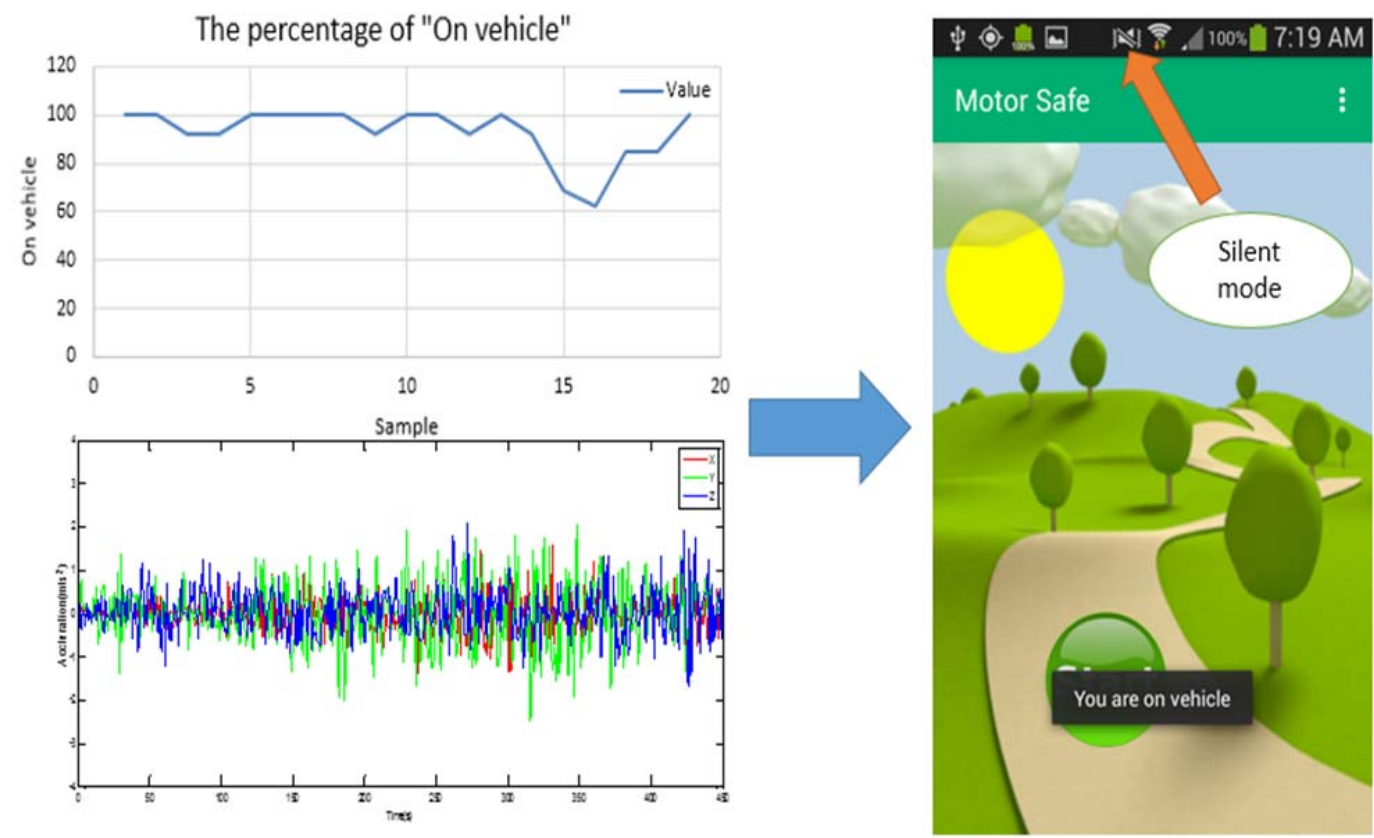

Figure 12. Change to silent mode while on vehicle

\section{B. Detecting accidents}

Figure 13 shows acceleration values in three axes for testing of the walking activity, the acceleration values in each axis change in short time intervals and between the moving statues.

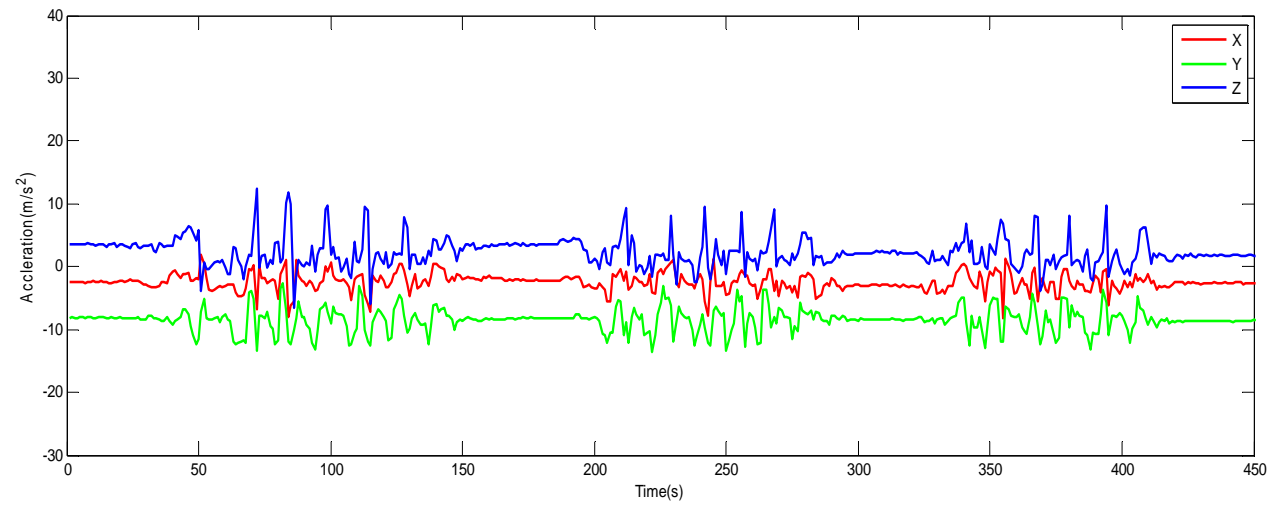

Fig. 13. Accelerometer data for the training of the walking activity

Figure 14 shows the data obtained from accelerometer for testing on vehicle with a stable speed, the value of acceleration in three axes have been changing fast between samples.

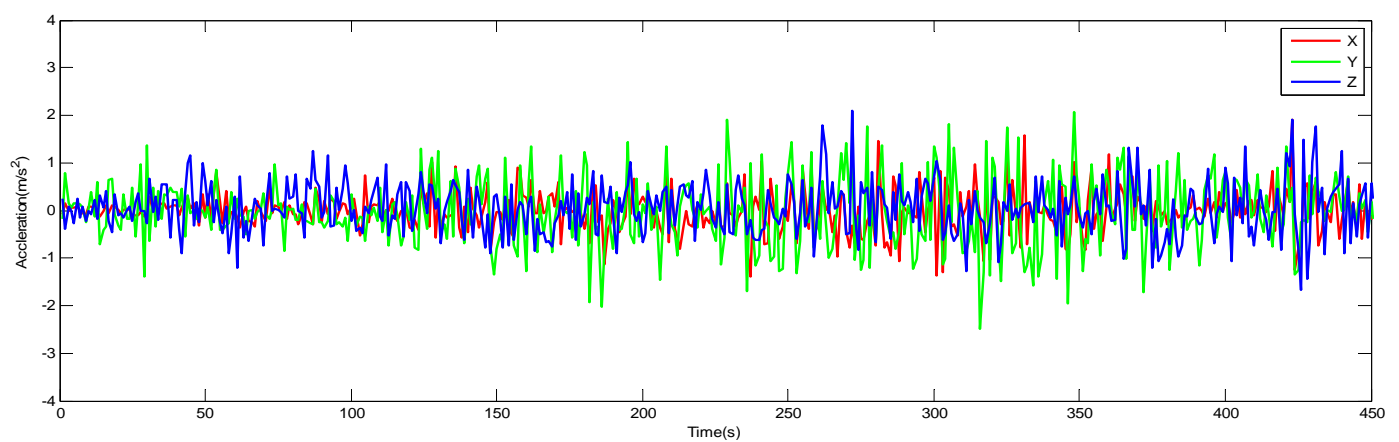

Fig. 14. Accelerometer data for training on the vehicle when no accident occurs 
As can be seen in Fig. 15, when the accident occurred, the values of acceleration changed suddenly.

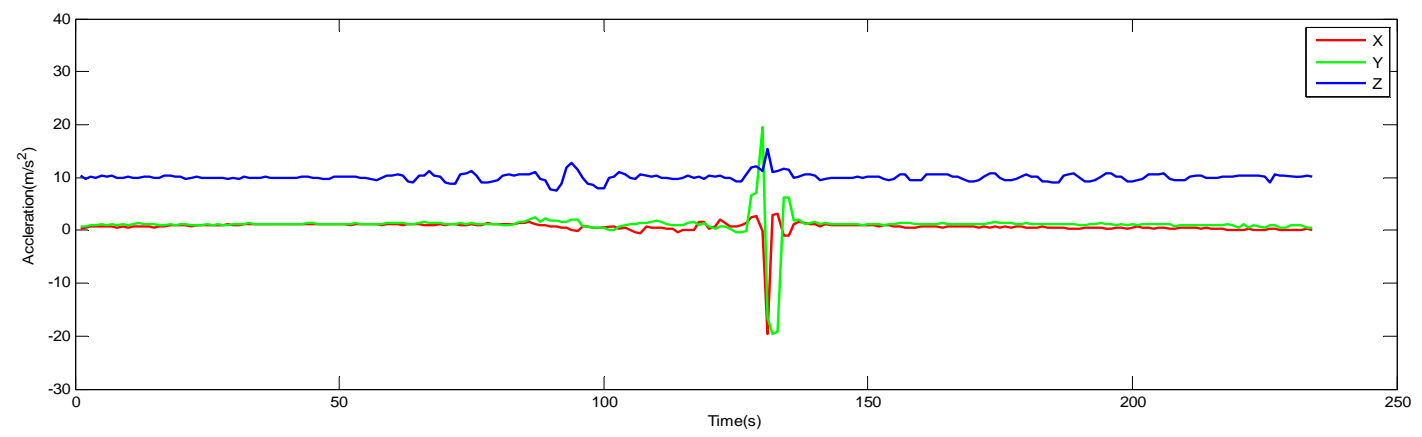

Fig. 15. Accelerometer data from demo accident

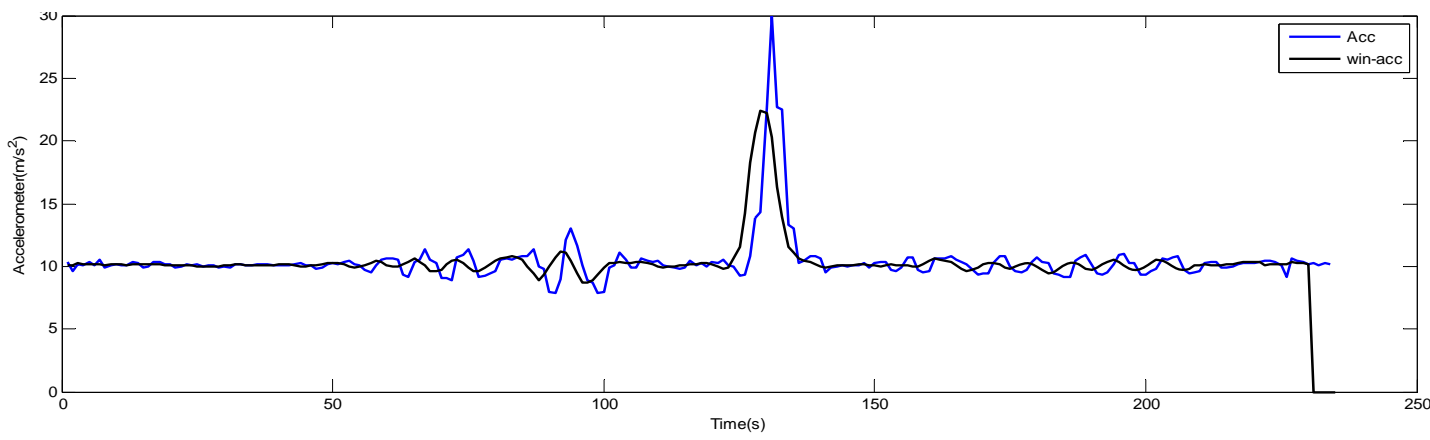

Fig. 16. Total acceleration (Acc) and mean (win-acc) with windowSize $=5$

Figure 16 shows the Acc, which is calculated by Equ. 3 and its average that is computed by using a movingaverage filter that is shown in Equ. 4. As can be seen in Fig. 16, when an accident occurs, both values of total acceleration and its mean increase suddenly. In order to confirm about the accident, an alert notification will appear on screen as shown in Fig. 17. If the user press on "No" button, there is no message will be send. If the user presses on "Confirm" button, a message will be sent to the user's relative person immediately. Without the confirmation, a message will sent to the user's relative person after 10 seconds.

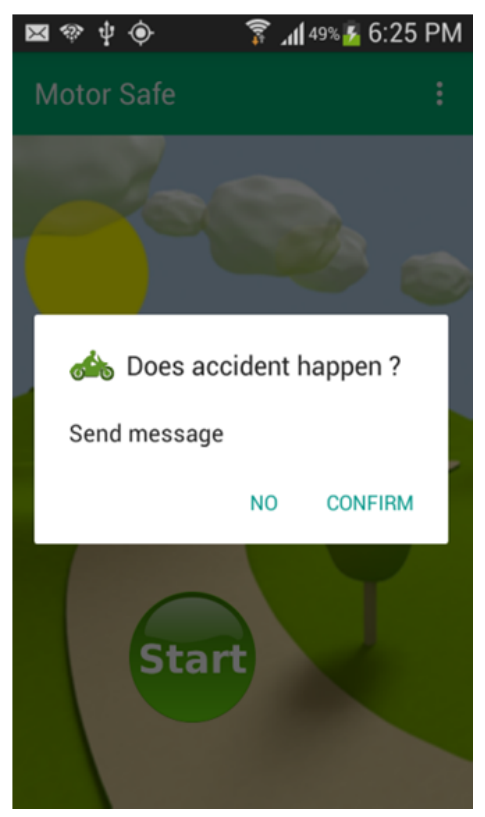

Figure 17. Notification when accident or free falling

Figure 18 shows the message send to the user' relative person. After receiving this message, the relative person just simply clicks to the link to see the accident location in the map. 


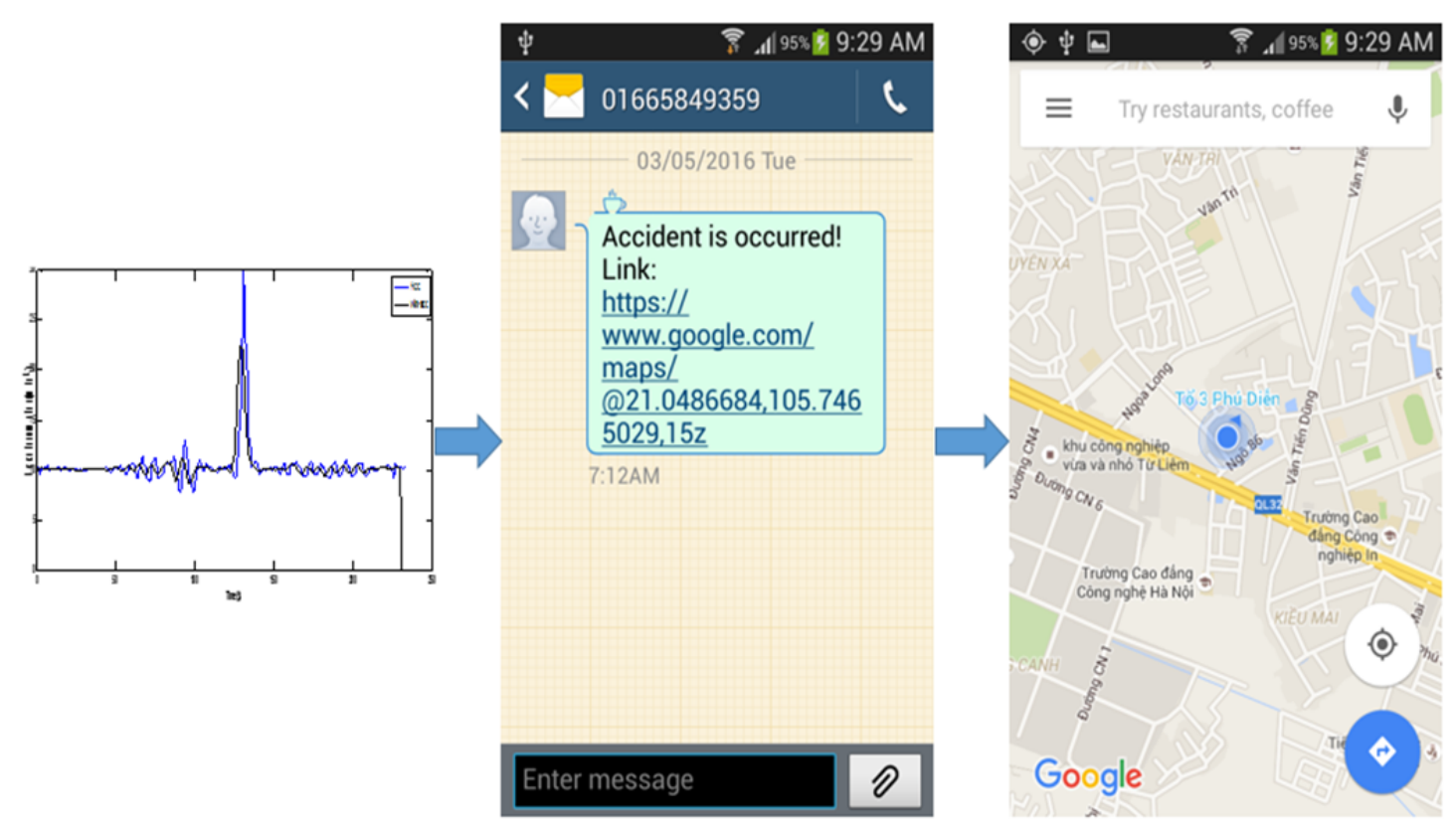

Fig. 18.Message notification and address of the accident

\section{IV.CONCLUSION}

In this paper, we have been successfully developed an application that can be installed on Android smartphones with important features such as: automatically detect the status of users and change the phone to silent mode if the users are driving; reject all unimportant and unknown calls with an SMS message except VIP contacts; provide a mechanism to receive emergency calls (even the caller is not in the VIP list), switch the phone to normal mode if the user stops using the motorbike. Furthermore, our application can detect an accident and automatically sends a message notification attached address location to the relatives and hospital services. This application is also installed and tested on many other Android phones. Through the app, the users can focus on driving safely. For the future research, we will make this application more friendly and improve the algorithm to increase the accuracy of our application.

\section{ACKNOWLEDGMENT}

This work has been supported by Ministry of Transport (Viet Nam) under Project No. DT174040.

\section{REFERENCES}

[1] Statistics of Accidents in Vietnam (2016). [Online]. Available:http://www.csgt.vn/tintuc/5711/Tai-nan-giao-thong-nam-2016-tiep-tucgiam-ca-ba-tieu-chi.html.

[2] Statistics of Accidents in Vietnam (2016). [Online]. Available: http://www.csgt.vn/tintuc/4653/Tinh-hinh-tai-nan-giao-thong-nam2015.html.

[3] Accidents Because of Pokemon Go Game (2016). [Online]. Available:http://tuoitre.vn/tin/chinh-tri-xa-hoi/20160819/tphcm-canh-baokhong-choi-pokemon-go-khi-lai-xe/1157803.html.

[4] Accidents Because of Using Mobile Phone in Controlling Vehicle Process (2017). [Online]. Available:http://giaothong.besaba.com/index.php?language=vi\&nv=news\&op=Huong-dan-an-toan-giao-thong/Su-dung-dien-thoaikhi-tham-gia-giao-thong-nguy-hiem-khon-luong-51.

[5] William M. Evanco, The Impact of Rapid Incident Detection on Freeway Accident Fatalities, Center for Information Systems, McLean,Virginia, 1996.

[6] Deepak Punetha, Deepak Kumar, Vartika Mehta, "Design and Realization of the Accelerometer based Transportation System", International Journal of Computer Applications,ISSN 0975 - 8887, Vol. 49, No. 15,pp. 17-20, July 2012.

[7] Accident Avoidance and Driver Assist Technologies. [Online]. Available:https://corporate.ford.com/microsites/sustainability-report2013-14/vehicle-avoidance.html.

[8] Active Safety - Warning, Supporting, Acting: Accident Prevention through Assistance Systems. [Online]. Available: http://media.daimler.com.

[9] Google APIs for Android, November 5, 2015. [Online]. Available:https://developers.google.com

[10] Shuangquan Wang, Canfeng Chen, Jian Ma, "Accelerometer based transportation mode recognition on mobile phones", Asia-Pacific Conference on Wearable Computing Systems, 2010, pp. 44-46.

[11] Bruno Fernandes, Vitor Gomes, Joaquim Ferreira and Arnaldo Oliveira, "Mobile Application for Automatic Accident Detection and Multimodal Alert", Vehicular Technology Conference (VTC Spring), 2015, IEEE, pp. 1-5.

[12] C. Thompson, J. White, B. Dougherty, A. Albright, and D. C. Schmidt, "Using smartphones to detect car accidents and provide situational awareness to emergency responders," MOBILWARE, 2010, pp. 29-42.

[13] J. Z. C. T. C. Juan Carlos Cano, Pietro Manzoni, "Providing Accident Detection in Vehicular Networks Through OBD-II Devices and AndroidbasedSmartphones", the 5th IEEE Workshop On User Mobility and Vehicular Networks, 2011, pp. 813-819.

[14] Miao W. (Intel), "Developing Sensor Applications on Intel ${ }^{\circledR}$ Atom ${ }^{\mathrm{TM}}$ Processor-Based Android* Phones and Tablets", Added September 26, 2013. 
[15] ÁdámZsolt Nagy, GáborFehér, BalázsLajtha, TamásSzincsák, "User activity recognition on mobile clients for Smart Public Transport Services", International Conference and Exhibition on Future RFID Technologies, Hungary, November 5-7, 2014. pp. $135-142$.

[16] Duc Ngoc Tran, DuyDinhPhan, "Human Activities Recognition in Android Smartphone Using Support Vector Machine", $7^{\text {th }}$ International Conference on Intelligent Systems, Modelling and Simulation, 2016, Thailand, pp. 64-68.

[17] Xing Su, Hanghang Tong, and Ping Ji, "Activity Recognition with Smartphone Sensors", Tsinghua science and technology, Vol. 19, No. 3, pp. 235-249,June 2014.

[18] Muhammad Shoaib, Stephan Bosch, OzlemDurmazIncel, Hans Scholten and Paul J.M. Havinga, "A Survey of Online Activity Recognition Using Mobile Phones",Sensors, Vol. 15, No. 1, pp. 2059-2085, 2015.

[19] Emmanuel Agu, Lecture 12: Activity Recognition, CS 403X Mobile and Ubiquitous Computing.

[20] KetanParmar, Android ActivityRecognition Example, Aug 27, 2015

[21] Rawan T. Khalil, Ala' F. Khalifeh, Khalid A. Darabkh, "Mobile-Free Driving with Android Phones: System Design and Performance Evaluation", 9th International Multi-Conference on Systems, Signals and Devices, 2012, pp. 1-6.

[22] Derick A. Johnson and Mohan M. Trivedi, "Driving Style Recognition Using a Smartphone as a Sensor Platform", 14th International IEEE Conference on Intelligent Transportation Systems, Washington DC, USA, October 5-7, 2011, pp. 1609-1615.

[23] Otebolaku, A. M., \& Andrade, M. T. "User context recognition using smartphonesensors and classification models", Journal of Network and Computer Applications, Vol. 66, pp. 33-51, 2016.

[24] Engelbrecht, J., Booysen, M., T.,vanRooyen, G. J., \&Bruwer, F. J., "Survey of smartphone-based sensing in vehicles for intelligent transportation system applications", IET Intelligent Transport Systems, Vol. 9, No. 10, pp. 924-935, 2015.

\section{AUTHOR PROFILE}

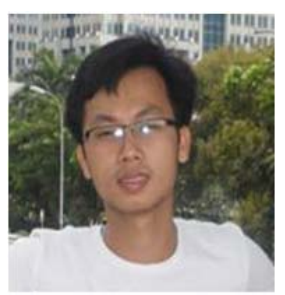

Pham Van Thanh was born in 1990. He received the bachelor degree in Electronics and Telecommunication at University of Engineering and Technology (UET) in 2012. He is currently a Master's student in Electronics and Telecommunication at UET and assistant lecturer in the Department of Fire protection systems,. Faculty of Automation and Technical equipment of Fire fighting\& Prevention, University of Fire Fighting and Prevention (UFFP). His research areas of interest include: Signal and image processing, personal safety equipments, Firefighter supporting devices, fire protection systems

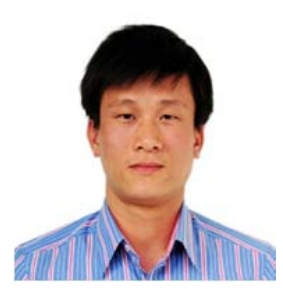

Anh T. Nguyen received the BSc degree in Physics and MSc degree in ElectronicsCommunication Technology from Vietnam National University, Hanoi (VNU) in 2003 and in 2007, respectively. He completed his Dr.-Eng degree from the Laboratory for Sensors, IMTEK, University of Freiburg, Germany in 2015. Currently, he is working at the Physical-Chemical Department, Le Quy Don Technical University, Ha Noi, Viet Nam. His research interests include cell-based impedance sensors, microfluidics, single cell analysis, and microcantilever.

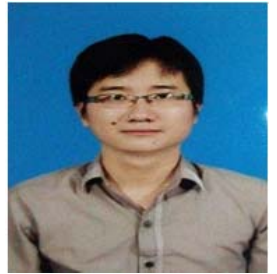

Tran DucNghia was born in 1986. He received his B.Sc and M.Sc degrees respectively in 2010, and 2015 at the Vietnam Maritime University (VIMARU), and the University of Engineering and Technology (UET), Vietnam National University - Hanoi (VNUH). He is currently a researcher at the Institute of Information Technology (IOIT), Vietnam Academy of Science and Technology (VAST). He has been participating in the implementation of some projects in information processing. He is the author and coauthor of some papers on Biomedical engineering and Bioinformatics. His present research interest is in mathematics and signal processing applications.

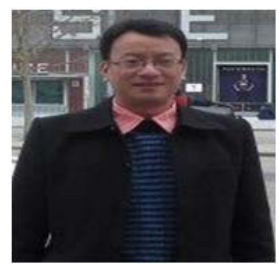

Nguyen DucAnh was born in 1977. He received his Bachelor and Master degrees at Le Quy Don University respectively in 2008, 2010 and $\mathrm{PhD}$ degree at Academy of Military Science and Technology in 2016. He is the author and co-author of serveral papers on fire protection engineering, digital signal processing and automation.

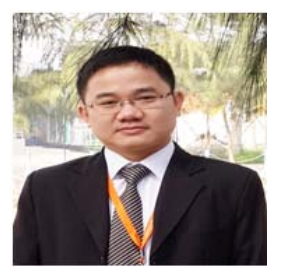

Tan D. Tran received his B.Sc, M.Sc, and PhD. degrees respectively in 2002, 2005, and 2010 at the University of Engineering and Technology (UET), Vietnam National University - Hanoi, Vietnam (VNUH), where he has been a lecturer since 2006. He was the recipient of the Vietnam National University, Hanoi, Vietnam Young Scientific Award in 2008. He is currently an Associate Professor with the Faculty of Electronics and Telecommunications, University of Engineering and Technology, Vietnam National University, Hanoi, Vietnam. He is the author and coauthor of many papers on MEMS based sensors and their application. His present research interest is in DSP applications. 